\title{
Optimal Investment and Reinsurance for Insurers with Uncertain Time-Horizon
}

\author{
Ailing Gu, ${ }^{1}$ Bo Yi, $^{2}$ and Dezhu $\mathrm{Ye}^{3}$ \\ ${ }^{1}$ Faculty of Applied Mathematics, Guangdong University of Technology, Guangzhou 510520, China \\ ${ }^{2}$ School of Mathematics and Computational Science, Sun Yat-sen University, Guangzhou 510275, China \\ ${ }^{3}$ Department of Finance and Research Institute of Finance, Jinan University, Guangzhou 510632, China \\ Correspondence should be addressed to Dezhu Ye; gzydz@126.com
}

Received 20 February 2014; Accepted 15 May 2014; Published 2 June 2014

Academic Editor: Pankaj Gupta

Copyright (c) 2014 Ailing Gu et al. This is an open access article distributed under the Creative Commons Attribution License, which permits unrestricted use, distribution, and reproduction in any medium, provided the original work is properly cited.

\begin{abstract}
This paper considers the investment-reinsurance problems for an insurer with uncertain time-horizon in a jump-diffusion model and a diffusion-approximation model. In both models, the insurer is allowed to purchase proportional reinsurance and invest in a risky asset, whose expected return rate and volatility rate are both dependent on time and a market state. Meanwhile, the market state described by a stochastic differential equation will trigger the uncertain time-horizon. Specifically, a barrier is predefined and reinsurance and investment business would be stopped if the market state hits the barrier. The objective of the insurer is to maximize the expected discounted exponential utility of her terminal wealth. By dynamic programming approach and FeynmanKac representation theorem, we derive the expressions for optimal value functions and optimal investment-reinsurance strategies in two special cases. Furthermore, an example is considered under the diffusion-approximation model, which shows some interesting results.
\end{abstract}

\section{Introduction}

Investment and reinsurance are two important ways for insurers to balance their profit and risk. Recently, the problem of optimal investment and/or reinsurance for insurers has been extensively studied in the literature. For example, in the framework of utility maximization, Bai and Guo [1], Cao and Wan [2], Irgens and Paulsen [3], and Liang et al. [4] discuss optimal proportional reinsurance-investment problems and Asmussen et al. [5], Gu et al. [6], and Zhang et al. [7] consider optimal excess-of-loss reinsurance-investment problems; in the framework of mean-variance, Bai and Zhang [8], Bäuerle [9], Li et al. [10], Zeng et al. [11], and Zeng and Li [12] study optimal investment and/or reinsurance problem.

However, the time-horizon in the literature mentioned above is assumed to be fixed. As we know many investors can be sure when they should enter the market, but they do not know certainly when their portfolio should be liquidated. Therefore, in the real market, fixed time-horizon is limited and unrealistic in some cases. For instance, the death of investors or some defaultable security defaults maybe terminate the horizon immediately. Based on these, it is of theoretical and practical interest to study optimal investment strategies under uncertain time-horizon. In fact, research on this subject has been literally discussed by some researchers in last decades: Karatzas and Wang [13] maximize the expected discount utility from consumption and the terminal wealth, assuming that the time-horizon is a stopping time with respect to (w.r.t.) an asset price; Kraf and Steffensen [14] study an optimal investment and consumption problem and introduce an arithmetic Brownian motion to trigger the exiting time. By maximizing the expected power utility of intermediate consumption and the terminal wealth, they obtain the optimal investment strategy; Blanchet-Scalliet et al. [15] obtain explicit expressions of the optimal strategy and the wealth process as a function of the distribution of the uncertain time-horizon in the case of CRRA utility functions; Zeng et al. [16] discuss the optimal investment and consumption strategies with state-dependent utility function under uncertain time-horizon. Recently, similar optimal 
problem is studied with mean-variance criterion, such as Kharroubi et al. [17], Guo and Cai [18], Yu [19], and Yao et al. [20].

Motivated by the above-mentioned work, we consider the optimal investment-reinsurance problems for an insurer with uncertain time-horizon, which is triggered by a market state described by a Brownian motion with drift. We assume that the insurer can invest her surplus in a risky asset (i.e., stock) and purchase proportional reinsurance to maximize the expected exponential utility of her terminal wealth. Two optimization problems are discussed in the cases of the surplus process described by a jump-diffusion model and a diffusion-approximation model. By adopting dynamic programming approach and Feynman-Kac representation theorem, we derive the optimal value functions and optimal investment-reinsurance strategies. We also find that the optimal investment strategies are time-varying and consist of a static Merton allocation and a dynamic hedging, but the optimal reinsurance strategies are constant. Finally, an example is provided in the diffusion-approximation model, which allows us to explicitly analyze the impacts of the market state and other parameters on the optimal investment strategy.

The rest of the paper is organized as follows. In Section 2, the model and the optimal investment-reinsurance problem with uncertain time-horizon are established. The optimal results in the jump-diffusion model and in the diffusionapproximation model are derived in Sections 3 and 4 . Moreover, an example is given to analyze the vital results in Section 5. The paper is concluded in Section 6.

\section{Model Formulation}

Let $\left(\Omega, \mathscr{F},\left\{\mathscr{F}_{t}: 0 \leq t \leq T\right\}, P\right)$ be a filtered complete probability space, where $T>0$ is a fixed predefined constant, representing the longest-decision time-horizon; $\left\{\mathscr{F}_{t}\right\}_{t \in[0, T]}$ is a filtration, satisfying the usual condition; that is, the filtration contains all $P$-null sets and is right continuous. All stochastic processes given in the following are assumed to be adapted on this space.

In a jump-diffusion model, the surplus process $\{X(t)\}$ of the insurer is given by

$$
d X(t)=c d t+\delta d W_{0}(t)-d \sum_{i=1}^{N(t)} Z_{i}
$$

where $c>0$ is a fixed constant premium rate; $\{N(t)\}$ is a homogeneous Poisson process with intensity $\lambda$, representing the number of claims which have arrived up to time $t$; the claim sizes $\left\{Z_{i}, i=1,2, \ldots\right\}$ are assumed to be independent and identically distributed (i.i.d.) positive random variables with common distribution $F$ with finite first-order moment $\mu_{\infty}=E\left(Z_{i}\right)$ and second-order moment $\sigma_{\infty}^{2}=E\left[Z_{i}^{2}\right]$; $\sum_{i=1}^{N(t)} Z_{i}$ represents the cumulative amount of claims up to time $t ;\left\{W_{0}(t)\right\}$ is one-dimensional Brownian motion and $\delta d W_{0}(t)$ represents stochastic uncertainty associated with the insurance business on the financial market. It is assumed that $\{N(t)\},\left\{Z_{i}, i=1,2, \ldots\right\}$, and $\left\{W_{0}(t)\right\}$ are independent of each other. Besides, the premium rate is calculated by the expected value principle; that is, $c=(1+\theta) \lambda \mu_{\infty}$, where $\theta>0$ is the relative safety loading of the insurer.

In this paper, the insurer is allowed to purchase proportional reinsurance or acquire new business to control insurance business risk. At any time $t \in[0, \tau]$, where $\tau$ is the investment horizon defined in the following, the retention level of proportional reinsurance is associated with a risk exposure $q(t) \in(0,+\infty)$. When $q(t) \in(0,1]$, it corresponds to a proportional reinsurance cover. Then, for each claim, the insurer should divert part of the premium to the reinsurer at the rate of $(1-q(t)) k$, where $k$ is the reinsurance premium rate of the reinsurer and calculated by the expected value principle; that is, $k=(1+\eta) \lambda \mu_{\infty}$, where $\eta$ is the relative safety loading of the reinsurer and $\eta \geq \theta$. If $\eta=\theta$, it shows that the reinsurance is cheap; if $\eta>\theta$, it shows that the reinsurance is not cheap; that is, the insurer needs to pay more premiums to the reinsurer than what she has received. Meanwhile, for each claim, the insurer only pays $100 q(t) \%$ while the rest $100(1-q(t)) \%$ is paid by the reinsurer. When $q(t) \in(1, \infty)$, the insurer acquires new business from other insurers as a reinsurer.

In addition to reinsurance, the insurer can invest her wealth in a risky asset, whose price process $\{S(t): t \geq 0\}$ satisfies the following differential equation:

$$
\frac{d S(t)}{S(t)}=\mu(t, y(t)) d t+\sigma(t, y(t)) d W(t), \quad S(0)=S_{0},
$$

where $\mu(t, y(t))>0$ and $\sigma(t, y(t))$ represent the expected instantaneous return rate and the volatility of return of the risky asset, respectively, and $y(t)$ is the market state variable, which satisfies the dynamics:

$$
d y(t)=\alpha(t, y(t)) d t+\beta(t, y(t)) d W_{1}(t), \quad y(0)=y_{0} .
$$

Here $\{W(t)\}$ and $\left\{W_{1}(t)\right\}$ are one-dimensional Brownian motions and related to correlation coefficient $\rho(\epsilon[-1,1])$. Denote $\bar{\tau}$ as a stopping time triggered by the market state variable $y(t)$. Therefore, for $T$ aforementioned, the investment horizon is $\tau=\bar{\tau} \wedge T$, where $\bar{\tau}=\inf \{s \geq t, y(s)=b\}, b>0$, is a predefined barrier with the initial state $y_{0} \leq b$.

For technical reasons we also assume that

(M1) $\mu(t, y)$ and $\sigma(t, y)$ are bounded functions of $t$ and $y$, and $\mu(t, y)$ is uniformly Hölder continuous;

(M2) $\alpha(t, y)$ and $\beta(t, y)$ are uniformly Lipschitz continuous.

Assumptions (M1) and (M2) can ensure that there exists a unique solution to (2) and (3).

Remark 1. We can understand that market state variable $y$ effects like an equity index. Generally, the equity index would increase while the market turns better; on the other hand, too high equity index perhaps indicates the generation of the bubble economy, which undermines the stability of the financial market. As a result, it is indispensable to make an 
upper bound for the market state variable $y$ for the sake of managing the financial market risk.

For each $t \in[0, \tau]$, let $b(t)$ be the proportion of total assets invested in the risky asset at time $t$. Define our control $\pi=\left(b^{\pi}(t), q^{\pi}(t)\right)$, a two-dimensional $\mathscr{F}_{t}$-predictable stochastic process, where $q^{\pi}(t)$ represents the retention level of reinsurance at time $t$. Thus, under the control strategy $\pi=\left(b^{\pi}(t), q^{\pi}(t)\right)$, the surplus process $X^{\pi}(t)$ of the insurer can be expressed by

$$
\begin{aligned}
d X^{\pi}(t)= & X^{\pi}(t) b^{\pi}(t)[\mu(t, y) d t+\sigma(t, y) d W(t)] \\
& +\left[c-k\left(1-q^{\pi}(t)\right)\right] d t \\
& +q^{\pi}(t) \delta d W_{0}(t)-q^{\pi}(t) d \sum_{i=1}^{N(t)} Z_{i} \\
= & X^{\pi}(t) b^{\pi}(t)[\mu(t, y) d t+\sigma(t, y) d W(t)] \\
& +q^{\pi}(t) \delta d W_{0}(t)+\lambda \mu_{\infty}\left(\theta-\eta+q^{\pi}(t)(1+\eta)\right) d t \\
& -q^{\pi}(t) d \sum_{i=1}^{N(t)} Z_{i} .
\end{aligned}
$$

Definition 2. A strategy $\pi$ is said to be admissible, if $\forall t \in[0, \tau],\left(b^{\pi}(t), q^{\pi}(t)\right)$ is $\mathscr{F}_{t}$-predictable with $b^{\pi}(t) \in$ $(-\infty,+\infty), q^{\pi}(t) \in(0,+\infty)$, and $E\left[\int_{t}^{\tau}\left(b^{\pi}(s)\right)^{2}+\left(q^{\pi}(s)\right)^{2} d s\right]<$ $\infty$, and (4) has a unique (strong) solution. Denote by $\Pi$ the set of all admissible strategies.

In this paper, the objective of the insurer is assumed to maximize her expected utility of the terminal wealth $X(\tau)$. Further, suppose that the insurer has an exponential utility function (see $[1,3])$ :

$$
U(x)=-\frac{1}{\gamma} \exp \{-\gamma x\}
$$

where $\gamma>0$ is the coefficient of risk aversion. For admissible strategy $\pi=\left(b^{\pi}(t), q^{\pi}(t)\right)$, the optimal value function with initial state $(x, y)$ at time $t$ is defined as

$$
V(t, x, y)=\max _{\pi \in \Pi} E^{t, x, y}\left[-\frac{1}{\gamma} \psi(\tau, y(\tau)) \exp \{-\gamma x(\tau)\}\right]
$$

with boundary condition

$$
\begin{aligned}
V(T, x, y) & =-\frac{1}{\gamma} \psi(T, y) \exp \{-\gamma x\}, \\
V(t, x, b) & =-\frac{1}{\gamma} \psi(t, b) \exp \{-\gamma x\},
\end{aligned}
$$

where $\psi(\cdot, \cdot)$, representing the subjective discount factor, is a positive function of $(t, y)$ (see Kraft and Steffensen [14]).

Remark 3. For technical reasons we do not consider risk-free asset and only discuss the case that the insurer invest her wealth in one risky asset. In fact, it is practicable if the insurer invests in the risky asset from time 0 to time $\tau$ and can invest all her wealth in the risk-free asset from time $\bar{\tau}$ to time $T$. For this purpose, we can take $\psi(\tau, y(\tau))=I_{\{\bar{\tau}<T\}} e^{\gamma r_{0}(T-\tau)}+I_{\{\bar{\tau} \geq T\}}$ with a positive constant $r_{0}$ standing for the risk-free interest rate. When $\bar{\tau}<T$, our aim is to maximize the terminal wealth at time $T$, and the insurer is forced to put all her wealth into the bank with risk-free interest rate $r_{0}$ from time $\tau$ to $T$; when $\bar{\tau} \geq T$, our aim is to maximize the terminal wealth at $\tau=T$.

\section{Optimal Results in the Jump-Diffusion Model}

In this section, we discuss problem (6) under the jumpdiffusion model. By dynamic programming approach and Feynman-Kac representation, we derive the expression for optimal investment and reinsurance strategy.

Similar to Fleming and Soner [21], the Hamilton-JacobiBellman equation (HJB) for problem (6) subject to (4) can be derived as follows:

$$
\begin{aligned}
\max _{\pi \in \Pi}\left\{V _ { x } ( t , x , y ) \left[x b^{\pi}(t) \mu(t, y)\right.\right. & \left.+\lambda \mu_{\infty}\left(\theta-\eta+q^{\pi}(t)(1+\eta)\right)\right] \\
+ & \frac{1}{2} \beta^{2}(t, y) V_{y y}(t, x, y) \\
+ & \frac{1}{2} V_{x x}(t, x, y)\left[\left(b^{\pi}(t)\right)^{2} x^{2} \sigma^{2}(t, y)+\left(q^{\pi}(t)\right)^{2} \delta^{2}\right] \\
+ & x b^{\pi}(t) \sigma(t, y) \beta(t, y) \rho V_{x y}(t, x, y) \\
+ & \alpha(t, y) V_{y}(t, x, y)+V_{t}(t, x, y) \\
+ & \left.\lambda E\left[V\left(t, x-q^{\pi}(t) Z, y\right)-V(t, x, y)\right]\right\}=0
\end{aligned}
$$

where $V_{t}, V_{x}, V_{y}, V_{x y}, V_{x x}$, and $V_{y y}$ are the first- and secondorder partial derivatives with respect to the corresponding variables.

In order to solve problem (6), we conjecture that the structure of the solution to (8) has the form

$$
W(t, x, y)=-\frac{1}{\gamma} g(t, y) \exp \{-\gamma x\}, \quad g(t, y)>0
$$

Direct calculation yields

$$
\begin{gathered}
W_{t}(t, x, y)=g_{t} \bar{W}, \quad W_{x}(t, x, y)=-\gamma g \bar{W}, \\
W_{x x}(t, x, y)=\gamma^{2} g \bar{W}, \quad W_{y}(t, x, y)=g_{y} \bar{W}, \\
W_{y y}(t, x, y)=g_{y y} \bar{W}, \\
E\left[W\left(t, x-q^{\pi}(t) Z, y\right)-W(t, x, y)\right]=E\left[e^{\gamma q^{\pi}(t) Z}-1\right] g \bar{W}, \\
W_{x y}(t, x, y)=-\gamma g_{y} \bar{W}, \quad \bar{W}=-\frac{1}{\gamma} \exp \{-\gamma x\} .
\end{gathered}
$$


Differentiating with respect to $b^{\pi}(t)$ in (8) implies

$$
\begin{aligned}
& \mu(t, y) W_{x}(t, x, y)+x b^{\pi}(t) \sigma^{2}(t, y) W_{x x}(t, x, y) \\
& +\sigma(t, y) \beta(t, y) \rho W_{x y}(t, x, y)=0 .
\end{aligned}
$$

Then we have

$$
\begin{aligned}
b^{\pi}(t) & \\
& =-\frac{\mu(t, y) W_{x}(t, x, y)+\beta(t, y) \sigma(t, y) \rho W_{x y}(t, x, y)}{x \sigma^{2}(t, y) W_{x x}(t, x, y)} \\
& =\frac{\mu(t, y)}{\gamma x \sigma^{2}(t, y)}+\frac{\rho \beta(t, y) g_{y}}{x \gamma \sigma(t, y) g} .
\end{aligned}
$$

Putting (12) and (10) into (8) yields that

$$
\begin{aligned}
\max _{q^{\pi}(t) \in(0, \infty]}\left\{g_{t}\right. & -\gamma \lambda \mu_{\infty}\left(\theta-\eta+q^{\pi}(t)(1+\eta)\right) g \\
& +\frac{1}{2} \gamma^{2}\left(q^{\pi}(t)\right)^{2} \delta^{2} g+\alpha(t, y) g_{y}+\frac{1}{2} \beta^{2}(t, y) g_{y y} \\
& +\lambda E\left[e^{\gamma q^{\pi}(t) Z}-1\right] g-\frac{\mu^{2}(t, y)}{2 \sigma^{2}(t, y)} g \\
& \left.-\frac{\rho \mu(t, y) \beta(t, y) g_{y}}{\sigma(t, y)}-\frac{\rho^{2} \beta^{2}(t, y) g_{y}^{2}}{2 g}\right\}=0 .
\end{aligned}
$$

According to the first condition with respect to $q^{\pi}(t)$ in (13), we have

$$
-(1+\eta) \lambda \mu_{\infty}+q^{\pi}(t) \delta^{2} \gamma+\lambda E\left[Z e^{\gamma q^{\pi}(t) Z}\right]=0 .
$$

To derive the solution of (14), we define

$$
f(q)=-(1+\eta) \lambda \mu_{\infty}+q \delta^{2} \gamma+\lambda E\left[Z e^{\gamma q Z}\right] .
$$

The first-order derivative of $f(q)$ shows

$$
f^{\prime}(q)=\gamma \delta^{2}+\lambda E\left[Z^{2} \gamma e^{\gamma q Z}\right]>0 .
$$

Taking into consideration that $f(0)<0$ and $\lim _{q \longrightarrow+\infty} f(q)>$ 0 , we know that there exists a unique $q^{\pi^{*}}(t) \in(0, \infty)$ such that $f\left(q^{\pi^{*}}(t)\right)=0$. Inserting $q^{\pi^{*}}(t)$ into (13), we obtain

$$
\begin{aligned}
g_{t}+\alpha(t, y) g_{y}+\frac{1}{2} \beta^{2}(t, y) g_{y y}-\frac{\rho \mu(t, y) \beta(t, y) g_{y}}{\sigma(t, y)} \\
-\frac{\rho^{2} \beta^{2}(t, y) g_{y}^{2}}{2 g}+J_{1}(t, y) g=0, \quad t \in[0, \tau], y \leq b,
\end{aligned}
$$

where

$$
\begin{aligned}
J_{1}(t, y)= & \lambda E\left[e^{\gamma q^{\pi^{*}}(t) Z}-1\right]-\frac{\mu^{2}(t, y)}{2 \sigma^{2}(t, y)} \\
& -\gamma \lambda \mu_{\infty}\left(\theta-\eta+q^{\pi^{*}}(t)(1+\eta)\right) \\
& +\frac{1}{2} \gamma^{2}\left(q^{\pi^{*}}(t)\right)^{2} \delta^{2} .
\end{aligned}
$$

Theorem 4. Assume that a positive solution to (17) exists. Under the jump-diffusion model (1), the optimal value function for problem (6) is given by

$$
V(t, x, y)=W(t, x, y)=-\frac{1}{\gamma} g(t, y) \exp \{-\gamma x\},
$$

the optimal investment strategy is given by

$$
b^{\pi^{*}}(t)=\frac{\mu(t, y)}{x \gamma \sigma^{2}(t, y)}+\frac{\rho \beta(t, y) g_{y}}{x \gamma \sigma(t, y) g},
$$

and the optimal reinsurance strategy $q^{\pi^{*}}(t)$ is determined by (14), where $g(t, y)$ is given by (17).

The proof is similar to that of Theorem 4 in Kraft and Steffensen [14]; hence it is omitted here.

Remark 5. The optimal investment strategy consists of two terms: the first term is an original optimal strategy as if the investment opportunities are constant for the insurer; the second term hedges the deviation from the speculative position due to the time-varying investment opportunities.

As we know that it is difficult to derive the explicit solution to (17) without other conditions, therefore in the following we consider two special cases which ensure that the solution to (17) exists and render the structures of the optimal investment-reinsurance strategies brief enough for our results analysis.

3.1. Case 1: $\rho=0$. If $\rho=0$, that is, $\{W(t)\}$ is independent of $\left\{W_{1}(t)\right\}$, simplify (17) to

$$
g_{t}+\alpha(t, y) g_{y}+\frac{1}{2} \beta^{2}(t, y) g_{y y}+J_{1}(t, y) g=0 .
$$

According to Feynman-Kac representation theorem (see Theorem 9.44 in Pascucci [22]) or Theorem 5.1 in Friedman [23], we derive the expression for $g(t, y)$ as follows:

$$
g(t, y)=E^{t, y}\left[\exp \left\{\int_{t}^{\tau} J_{1}(s, y(s)) d s\right\} \psi(\tau, y(\tau))\right] .
$$

As a result, Corollary 6 is given as follows.

Corollary 6. In the jump-diffusion model, when $\rho=0$, the optimal value function for problem (6) is given by

$$
V(t, x, y)=-\frac{1}{\gamma} g(t, y) \exp \{-\gamma x\}
$$


with $g(t, y)=E^{t, y}\left[\exp \left\{\int_{t}^{\tau} J_{1}(s, y) d s\right\} \psi(\tau, y(\tau))\right]$, and the optimal investment strategy

$$
b^{\pi^{*}}(t)=\frac{\mu(t, y)}{x \gamma \sigma^{2}(t, y)}
$$

and optimal reinsurance strategy $q^{\pi^{*}}(t)$ is determined by (14).

In this case, no hedging term appears in the optimal investment strategy due to the constant investment opportunity set.

3.2. Case 2: $\rho=1$ and $\mu(t, y)=\mu(t), \sigma(t, y)=\sigma(t)$. In this case, $g(t, y)$ in (17) has the form $g(t, y)=e^{h(t, y)}$. Therefore, $h(t, y)$ satisfies the following differential equation:

$$
\begin{aligned}
& h_{t}+h_{y}\left[\alpha(t, y)-\frac{\mu(t)}{\sigma(t)} \beta(t, y)\right]+\frac{1}{2} \beta^{2}(t, y) h_{y y} \\
& +R_{1}(t)=0, \quad t \in[0, \tau], y \leq b,
\end{aligned}
$$

where

$$
\begin{aligned}
R_{1}(t)= & \lambda E\left[e^{\gamma q^{\pi^{*}}(t) Z}-1\right]-\frac{\mu^{2}(t)}{2 \sigma^{2}(t)} \\
& -\gamma \lambda \mu_{\infty}\left(\theta-\eta+q^{\pi^{*}}(t)(1+\eta)\right) \\
& +\frac{1}{2} \gamma^{2}\left(q^{\pi^{*}}(t)\right)^{2} \delta^{2} .
\end{aligned}
$$

In order to derive the expression of $h(t, y)$, we define a new measure (since $\mu(t)$ and $\sigma(t)$ are deterministic functions, one could obviously verify that the Novikov condition is satisfied, which implies that $Q$ is indeed a new equivalent probability measure) $Q$ :

$$
\frac{d Q}{d P}=\exp \left\{-\int_{0}^{t} \frac{\mu(t)}{\sigma(t)} d W_{1}(t)-\frac{1}{2} \int_{0}^{t} \frac{\mu^{2}(t)}{\sigma^{2}(t)} d t\right\}
$$

such that $\widehat{W}_{1}(t)=\int_{0}^{t}(\mu(s) / \sigma(s)) d s+W_{1}(t)$ and

$$
d y=\left(\alpha(t, y)-\frac{\mu(t)}{\sigma(t)} \beta(t, y)\right) d t+\beta(t, y) d \widehat{W}_{1}(t)
$$

Based on Feynman-Kac representation theorem, we have

$$
h(t, y)=E_{t, y}^{Q}\left[\int_{t}^{\tau} R_{1}(s) d s+\ln \psi(\tau, y(\tau))\right],
$$

where $E_{t, y}^{\mathrm{Q}}$ denotes the expectation under measure $Q$ with the market state $y$ at time $t$. According to (20), the optimal investment strategy is given by

$$
b^{\pi^{*}}(t)=\frac{\mu(t)}{x \gamma \sigma^{2}(t)}+\frac{\beta(t, y) h_{y}}{x \gamma \sigma(t)} .
$$

Summarizing the above discussion, we have the following corollary.
Corollary 7. In the jump-diffusion model, when $\rho=1$ and $\mu(t, y)=\mu(t), \sigma(t, y)=\sigma(t)$, the optimal value function for problem (6) is given by

$$
V(t, x, y)=-\frac{1}{\gamma} \exp \{-\gamma x+h(t, y)\}
$$

the optimal investment strategy is given by (30), and the optimal reinsurance strategy $q^{\pi^{*}}(t)$ is determined by (14), where $h(t, y)$ is given by (29).

Remark 8. The optimal investment strategy can be separated to two components: the first component $\mu(t) / x \gamma \sigma^{2}(t)$ can be called the myopic term which is independent of time-varying market state. The second component $\beta(t, y) h_{y} / x \gamma \sigma(t)$ is a hedging term correlated with the market state process. It should disappear if decision horizon is fixed, which implies that this term affects hedging device for the time-varying market state.

\section{Optimal Results in the Diffusion-Approximation Model}

In this section, we discuss the solution to problem (6) for an insurer whose surplus process is approximated by a diffusion process.

We assume that the aggregate claim process in (1) is described by a Brownian motion with drift; that is,

$$
C(t)=\alpha_{0} t-\sigma_{0} W_{2}(t)
$$

where $\left\{W_{2}(t)\right\}$ is a Brownian motion and independent of $\{W(t)\},\left\{W_{0}(t)\right\}$, and $\left\{W_{1}(t)\right\}$. According to Grandell [24], the compound Poisson process can be approximated by a diffusion process; then

$$
\sum_{i=1}^{N(t)} Z_{i} \approx C(t)=\lambda \mu_{\infty} t-\sqrt{\lambda} \sigma_{\infty} W_{2}(t)
$$

where $\lambda \mu_{\infty}=\alpha_{0}, \sqrt{\lambda} \sigma_{\infty}=\sigma_{0}$ (see $\left.[4,25]\right)$. Then the surplus process (1) can be approximated by

$$
d X_{\infty}(t)=\theta \lambda \mu_{\infty} d t+\delta d W_{0}(t)+\sqrt{\lambda} \sigma_{\infty} d W_{2}(t)
$$

Similar to Section 3, for each $t \in[0, \tau]$, define investmentreinsurance strategy $\bar{\pi}=\left(b^{\bar{\pi}}(t), q^{\bar{\pi}}(t)\right)$ and let $b^{\bar{\pi}}(t)$ be the proportion of total assets invested in the risky asset at time $t$ and $q^{\bar{\pi}}(t)$ represents the retention level of reinsurance at time $t$. Thus, under the control strategy $\bar{\pi}=\left(b^{\bar{\pi}}(t), q^{\bar{\pi}}(t)\right)$, the surplus process of the insurer can be expressed by

$$
\begin{aligned}
d X_{\infty}^{\bar{\pi}}(t)= & \lambda \mu_{\infty}\left(\theta-\eta+q^{\bar{\pi}} \eta\right) d t+\delta q^{\bar{\pi}}(t) d W_{0}(t) \\
& +\sqrt{\lambda} \sigma_{\infty} q^{\bar{\pi}}(t) d W_{2}(t) \\
& +X_{\infty}^{\bar{\pi}}(t) b^{\bar{\pi}}(t)[\mu(t, y) d t+\sigma(t, y) d W(t)] .
\end{aligned}
$$


Definition 9. A strategy $\bar{\pi}$ is said to be admissible, if $\forall t \in$ $[0, \tau],\left(b^{\bar{\pi}}(t), q^{\bar{\pi}}(t)\right)$ is $\mathscr{F}_{t}$-progressively measurable with $b^{\bar{\pi}}(t)$ $\in(-\infty,+\infty), q^{\bar{\pi}}(t) \in(0, \infty), E\left[\int_{t}^{\tau}\left(\left(b^{\bar{\pi}}(s)\right)^{2}+\left(q^{\bar{\pi}}(s)\right)^{2}\right) d s\right]<$ $\infty$, and (35) has a unique (strong) solution. Denote by $\bar{\Pi}$ the set of all admissible strategies.

Now, by similar method used in Section 3 we solve problem (6) subject to (35). Then the corresponding HJB equation is given by

$$
\begin{gathered}
\max _{\bar{\pi} \in \bar{\Pi}}\left\{V_{t}+V_{x}\left[\lambda \mu_{\infty}\left(\theta-\eta+q^{\bar{\pi}} \eta\right)+x b^{\bar{\pi}}(t) \mu(t, y)\right]\right. \\
+\frac{1}{2} V_{x x}\left[\delta^{2}\left(q^{\bar{\pi}}(t)\right)^{2}+\lambda \sigma_{\infty}^{2}\left(q^{\bar{\pi}}(t)\right)^{2}\right. \\
\left.+x^{2}\left(b^{\bar{\pi}}(t)\right)^{2} \sigma^{2}(t, y)\right] \\
+\alpha(t, y) V_{y}+\frac{1}{2} \beta^{2}(t, y) V_{y y} \\
\left.+\rho x b^{\bar{\pi}}(t) \sigma(t, y) \beta(t, y) V_{x, y}\right\}=0
\end{gathered}
$$

with boundary condition (7).

We assume that (36) has a solution $W(t, x, y)$ with the same structure of (9). Then by the first-order condition for HJB equation (36) we have optimal investment-reinsurance strategy:

$$
\begin{gathered}
b^{\bar{\pi}^{*}}(t)=-\frac{\mu(t, y) W_{x}}{x \sigma^{2}(t, y) W_{x x}}-\frac{\rho \beta(t, y) W_{x y}}{x \sigma(t, y) W_{x x}}, \\
q^{\bar{\pi}^{*}}(t)=-\frac{\eta \lambda \mu_{\infty} W_{x}}{\left(\delta^{2}+\lambda \sigma_{\infty}^{2}\right) W_{x x}} .
\end{gathered}
$$

Putting (10) and (37) into (36) yields

$$
\begin{aligned}
g_{t}+\alpha(t, y) g_{y}+\frac{1}{2} \beta^{2}(t, y) g_{y y}-\frac{\rho \mu(t, y) \beta(t, y) g_{y}}{\sigma(t, y)} \\
-\frac{\beta^{2}(t, y) g_{y}^{2} \rho^{2}}{2 g}+J_{2}(t, y) g=0,
\end{aligned}
$$

where $J_{2}(t, y)=(\eta-\theta) \gamma \lambda \mu_{\infty}-\left(\eta^{2} \lambda^{2} \mu_{\infty}^{2} / 2\left(\delta^{2}+\lambda \sigma_{\infty}^{2}\right)\right)-$ $\mu^{2}(t, y) / 2 \sigma^{2}(t, y)$. By the similar analysis as Section 3 , we acquire the following theorem.

Theorem 10. Assume that a positive solution to (38) exists. Under the diffusion-approximation model (35), the optimal value function for problem (6) is given by

$$
V(t, x, y)=W(t, x, y)=-\frac{1}{\gamma} g(t, y) \exp \{-\gamma x\},
$$

the optimal investment strategy is

$$
b^{\bar{\pi}^{*}}(t)=\frac{\mu(t, y)}{x \gamma \sigma^{2}(t, y)}+\frac{\rho \beta(t, y) g_{y}}{x \gamma \sigma(t, y) g},
$$

and the optimal reinsurance strategy is

$$
q^{\bar{\pi}^{*}}(t)=\frac{\eta \lambda \mu_{\infty}}{\gamma\left(\delta^{2}+\lambda \sigma_{\infty}^{2}\right)},
$$

where $g(t, y)$ is given by (38).

According to Theorem 5.1 in Friedman [23], there exists positive solution to (38) in Cases 1 and 2 given in Section 3. Therefore, two corollaries can be derived as follows.

Corollary 11. In the diffusion-approximation model, when $\rho=0$, the optimal value function for problem (6) is given by

$$
V(t, x, y)=-\frac{1}{\gamma} g(t, y) \exp \{-\gamma x\},
$$

the optimal investment strategy is

$$
b^{\bar{\pi}^{*}}(t)=\frac{\mu(t, y)}{x \gamma \sigma^{2}(t, y)},
$$

and the optimal reinsurance strategy is

$$
q^{\bar{\pi}^{*}}(t)=\frac{\eta \lambda \mu_{\infty}}{\gamma\left(\delta^{2}+\lambda \sigma_{\infty}^{2}\right)}
$$

where $g(t, y)=E^{t, y}\left[\exp \left\{\int_{t}^{\tau} J_{2}(s, y(s)) d s\right\} \psi(\tau, y(\tau))\right]$ and $J_{2}(t, y)=(\eta-\theta) \gamma \lambda \mu_{\infty}-\left(\eta^{2} \lambda^{2} \mu_{\infty}^{2} / 2\left(\delta^{2}+\lambda \sigma_{\infty}^{2}\right)\right)-\mu^{2}(t, y) /$ $2 \sigma^{2}(t, y)$.

Corollary 12. In the diffusion-approximation model, when $\rho=1$ and $\mu(t, y)=\mu(t), \sigma(t, y)=\sigma(t)$, the optimal value function for problem (6) is given by

$$
V(t, x, y)=-\frac{1}{\gamma} \exp \{-\gamma x+h(t, y)\}
$$

and the optimal investment strategy and the optimal reinsurance strategy can be given by

$$
\begin{gathered}
b^{\bar{\pi}^{*}}(t)=\frac{\mu(t)}{x \sigma^{2}(t) \gamma}+\frac{\beta(t, y)}{\gamma x \sigma(t)} h_{y}, \\
q^{\bar{\pi}^{*}}(t)=\frac{\eta \lambda \mu_{\infty}}{\gamma\left(\delta^{2}+\lambda \sigma_{\infty}^{2}\right)},
\end{gathered}
$$

where $h(t, y)=E_{t, y}^{Q}\left[\int_{t}^{\tau} R_{2}(s) d s+\ln \psi(\tau, y(\tau))\right]$ and $R_{2}(t)=$ $(\eta-\theta) \gamma \lambda \mu_{\infty}-\left(\eta^{2} \lambda^{2} \mu_{\infty}^{2} / 2\left(\delta^{2}+\lambda \sigma_{\infty}^{2}\right)\right)-\mu^{2}(t) / 2 \sigma^{2}(t)$.

Remark 13. From Theorems 4 and 10, we find that the optimal investment strategy is time-varying, but the optimal reinsurance strategy is constant. This can be explained from two sides. On one hand, the optimal investment strategy only depends on the financial market and the parameters of financial market are time-varying. On the other hand, the optimal reinsurance strategy is independent of financial market and only depends on the insurance market. Moreover, the premium income rate of the insurer and the reinsurance 
premium rate are assumed to be constant and other parameters in the risk model of the insurer are not time-varying. In summary, the change of the surplus process of the insurer would not trigger the variation of the optimal investment strategy.

Remark 14. From Corollaries 6 and 11, the optimal investment strategies depend on the market state but have nothing to do with the exiting time. However, based on Corollaries 7 and 12, the optimal investment strategies can be decomposed to two parts. The first part is a myopic term and the second part is a dynamic hedging term which is correlated to the market state process and reflects that the optimal investment strategy would change with the probability distribution of exiting time.

\section{Example}

According to Corollaries 6-12, the optimal reinsurance strategy is constant and does not change with time; however, the optimal investment strategy is time-varying. Moreover, we find that the optimal investment strategy is dependent on the exiting time in Case 2, but not in Case 1. Therefore, for the sake of more comprehensive analysis, we give an example to explicitly show the optimal investment strategy in the diffusion-approximation model under Case 2 (similar example can be given in the jump-diffusion model) and analyze the impact of the market state and some parameters on the optimal investment strategy. Here, we assume that $\rho=1, \mu(t, y)=\mu, \sigma(t, y)=\sigma$, and the market state process $y(t)$ is driven by a geometric Brownian motion; that is,

$$
\frac{d y(t)}{y(t)}=\alpha d t+\beta d W_{1}(t)
$$

Therefore, under the new measure Q, defined in Section 3,

$$
d I(t)=\left(\alpha-\frac{1}{2} \beta^{2}-\frac{\beta \mu}{\sigma}\right) d t+\beta d \widehat{W}_{1}(t)
$$

where $I(t)=\ln y(t), \widehat{W}(t)=(\mu / \sigma) t-W_{1}(t)$. Furthermore, we assume that

$$
\psi(\tau, y)=I_{\{\bar{\tau}<T\}} e^{\gamma r_{0}(T-\tau)}+I_{\{\bar{\tau} \geq T\}},
$$

where $r_{0}$ is the risk-free interest rate. Let

$$
\widehat{\alpha}=\frac{\alpha-(1 / 2) \beta^{2}-\beta \mu / \sigma}{\beta}, \quad \delta(t, I)=\frac{\ln b-I(t)}{\beta} ;
$$

then the conditional Q-probability distribution of $\bar{\tau}$ can be given by (see Kraft and Steffensen [14] or Karatzas and Shreve [26])

$$
\begin{aligned}
\widehat{P}(\bar{\tau} \leq u \mid I(t)=I)= & \mathcal{N}\left(\frac{\widehat{\alpha}(u-t)-\delta(t, I)}{\sqrt{u-t}}\right) \\
& +e^{2 \widehat{\alpha} \delta(t, I)} \mathcal{N}\left(\frac{-\widehat{\alpha}(u-t)-\delta(t, I)}{\sqrt{u-t}}\right),
\end{aligned}
$$

where $\mathcal{N}(\cdot)$ is the distribution function of the standard normal distribution. According to the distribution of a function of a random vector, we have

$$
\begin{aligned}
\widehat{P}(\bar{\tau} \leq u \mid y(t)=y)= & \widehat{P}(\bar{\tau} \leq u \mid I(t)=\ln (y)) \\
= & \mathcal{N}\left(\frac{\widehat{\alpha}(u-t)-\delta(t, \ln (y))}{\sqrt{u-t}}\right) \\
& +e^{2 \widehat{\alpha} \delta(t, \ln (y))} \\
& \times \mathcal{N}\left(\frac{-\widehat{\alpha}(u-t)-\delta(t, \ln (y))}{\sqrt{u-t}}\right) .
\end{aligned}
$$

From Corollary 12, we obtain

$$
\begin{aligned}
h(t, y)= & E_{t, y}^{Q}\left[\int_{t}^{\tau} R_{2}(s) d s+\ln \psi(\tau, y(\tau))\right] \\
= & E_{t, y}^{Q}\left[\left(R_{2}(\tau-t)+\gamma r_{0}(T-\tau)\right) I_{\{\bar{\tau}<T\}}\right] \\
& +E_{t, y}^{Q}\left[R_{2}(T-t) I_{\{\bar{\tau} \geq T\}}\right] \\
= & \frac{\left(R_{2}-\gamma r_{0}\right) \delta(t, \ln (y))}{\widehat{\alpha}} \\
& \times\left[\mathcal{N}\left(\frac{\widehat{\alpha}(T-t)-\delta(t, \ln (y))}{\sqrt{T-t}}\right)\right. \\
& -\left(R_{2}-\gamma r_{0}\right)(T-t) \\
& \times\left[\mathcal{N}\left(\frac{\widehat{\alpha}(T-t)-\delta(t, \ln (y))}{\sqrt{T-t}}\right)\right. \\
& \left.-R_{2}^{2 \widehat{\alpha} \delta(t, \ln (y))} \mathcal{N}\left(\frac{-\widehat{\alpha}(T-t)-\delta(t, \ln (y))}{\sqrt{T-t}}\right)\right] \\
& \left.+e^{2 \widehat{\alpha} \delta(t, \ln (y))} \mathcal{N}\left(\frac{-\widehat{\alpha}(T-t)-\delta(t, \ln (y))}{\sqrt{T-t}}\right)\right]
\end{aligned}
$$

Based on the above calculation, the optimal investment strategy can be given by

$$
b^{\bar{\pi}^{*}}(t)=\frac{\mu}{x \sigma^{2} \gamma}+\frac{\beta y}{\gamma x \sigma} h_{y}
$$

where

$$
\begin{aligned}
h_{y}=\frac{\left(R_{2}-\gamma r_{0}\right)}{\beta y}\left[-\frac{1}{\widehat{\alpha}}\right. & \mathcal{N}\left(\frac{\widehat{\alpha}(T-t)-\delta(t, \ln (y))}{\sqrt{T-t}}\right) \\
& +\left(\frac{1}{\widehat{\alpha}}+2 \delta+2 \widehat{\alpha}(T-t)\right) \\
& \left.\times \mathcal{N}\left(\frac{-\widehat{\alpha}(T-t)-\delta(t, \ln (y))}{\sqrt{T-t}}\right)\right] \\
-2 \frac{\left(R_{2}-\gamma r_{0}\right)}{\beta y} f & \left(\frac{\widehat{\alpha}(T-t)-\delta(t, \ln (y))}{\sqrt{T-t}}\right),
\end{aligned}
$$




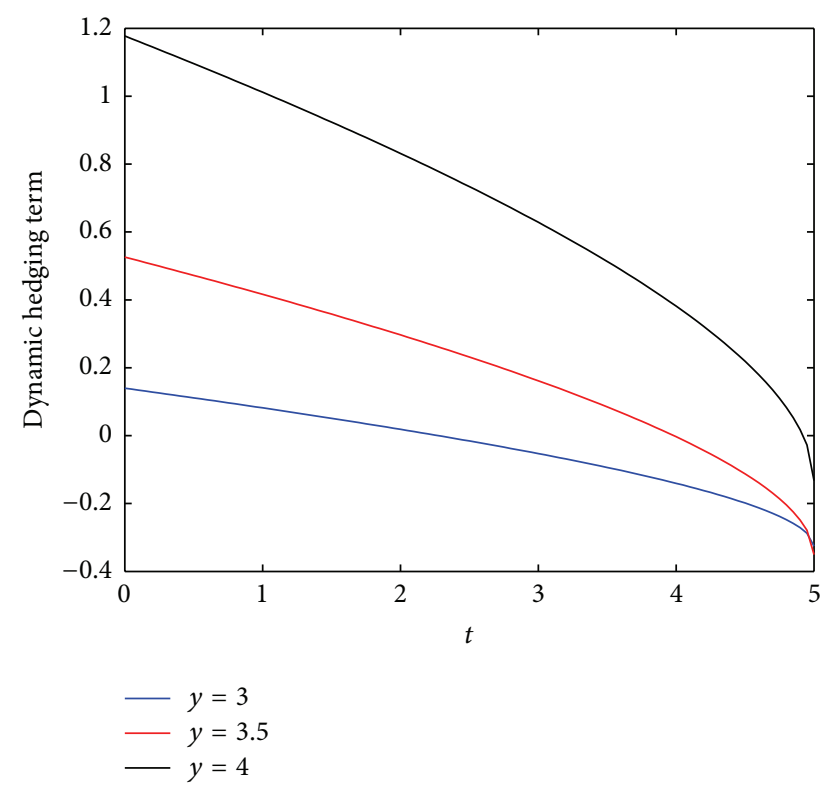

FIGURE 1: Evolution of the dynamic hedging term.

and $f(\cdot)$ represents the density function of the stand normal distribution.

According to our assumption, we obtain the explicit expression for the optimal investment strategy, which makes the sensitivity analysis possible in this example. In the following, the basic parameters are given by $x=1, T=5$, $\gamma=0.5, \mu_{\infty}=0.5, \sigma_{\infty}=1, \theta=0.15, \eta=0.38, r_{0}=0.05$, $\mu=0.12, \lambda=1, \sigma=0.25, \alpha=0.1, \beta=0.21, \delta=0.1$, and $b=5$.

In this paper, the market state variable $y$ is an important variable for it triggers the exiting time and its effects on the optimal investment strategy cannot be ignored. As shown in (54), the optimal investment strategy is made up of two terms. The first term is the static Merton allocation (myopic demand) and independent of $y$, while the second term is the dynamic hedging and depends on $y$. This term reflects the hedging demands against the risk of the uncertain exiting market. As is shown by Figure 1, the hedging term would increase with the market state, which stands for an increasing demand to hedge the financial market risk; but it progressively decreases with respect to time, which shows that the hedging demand shrinks throughout the timehorizon. This feature also implies that the hedging demand is much more important for the long-term insurer. It can be interpreted as the following perspective: the long-term insurer should have more concerns about the early-exitingmarket behavior, which leads to a higher demand on hedging. In general, the optimal investment strategy increases with $y$ and is larger than the Merton allocation before closing to the terminal time; see Figure 2(a).

Other parameters $\mu, \beta, \gamma$, and $\sigma$ also have nonignorable impacts on the optimal investment strategy. Specifically, the optimal investment strategy increases with $\mu$ and $\beta$. This result is reasonable as $\mu$ is the expected return rate. The insurer likes to invest more with the increase of return rate, but it seems like an anti-intuition result for $\beta$ which is the volatility of the market state. Figure 2(b) with $y=3$ illustrates this interesting phenomenon, which also implies that the insurer is prone to put more money in the risky asset to hedge the uncertain time-horizon under the situation that the market state fluctuates dramatically. This phenomenon can be interpreted as the following way: the increase of the market state's volatility will lead to a high chance to exit the market, which presents a short-term behavior of the insurer, that is, high-proportion-wealth allocation in risky asset.

Different from $\mu$ and $\beta$, the volatility of the risky asset $\sigma$ and the degree of risk aversion $\gamma$ have negative effects on the optimal investment strategy. Figure 2(c) provides instruction that the insurer should decrease the investment in risky asset with the increase of $\sigma$ and Figure 2(d) shows that the insurer would like to decrease the investment in the risky asset with $\gamma$ intuitively.

\section{Conclusion}

As we know the real market is not invariable but in a perpetual dynamic development. Thus it would be remarkably significant to study the optimal investment-reinsurance problem for an insurer with uncertain exiting time. In some worse scenario, the wise insurer should take into account the option to exit market for avoiding unaccepted risk. In this paper, we use Brownian motion with drift to describe the market state, which determines the exiting time. In addition to the investment in one risky asset, the insurer can purchase proportional reinsurance in a jump-diffusion model and a diffusion-approximation model, respectively. The insurer aims to maximize the expected discounted utility of her terminal wealth. By dynamic programming approach and Feynman-Kac representation theorem, we derived the optimal value functions and optimal strategies under both models. We find that the probability contribution of exiting time has an effect on the optimal strategies but has no effect on the optimal reinsurance strategy in the case of $\rho=1$. Moreover, the optimal investment strategy is timevarying and independent of the insurance business and vice versa. Finally, an example is given to show that the optimal investment strategy is considerably sensitive to the market risk, which buttresses the importance of the market risk in practical financial applications.

\section{Conflict of Interests}

The authors declare that there is no conflict of interests regarding the publication of this paper.

\section{Acknowledgments}

This research is supported by Grants from the National Natural Science Foundation of China (nos. 71231008 and 71201173), Humanity and Social Science Foundation of MOE of China (no. 13YJCZH247), National Science Foundation of Guangdong Province of China (no. S2013010011959), Foundation for Distinguished Young Talents in Higher Education of Guangdong, China (1012WYM-001), Fundamental 


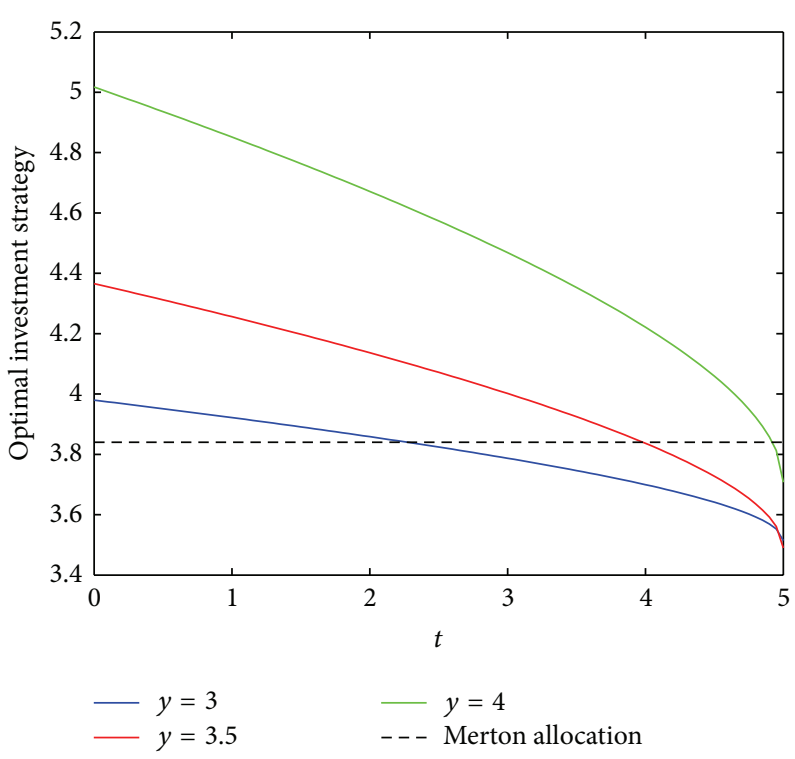

(a) The effect of $y$ on the optimal investment strategy

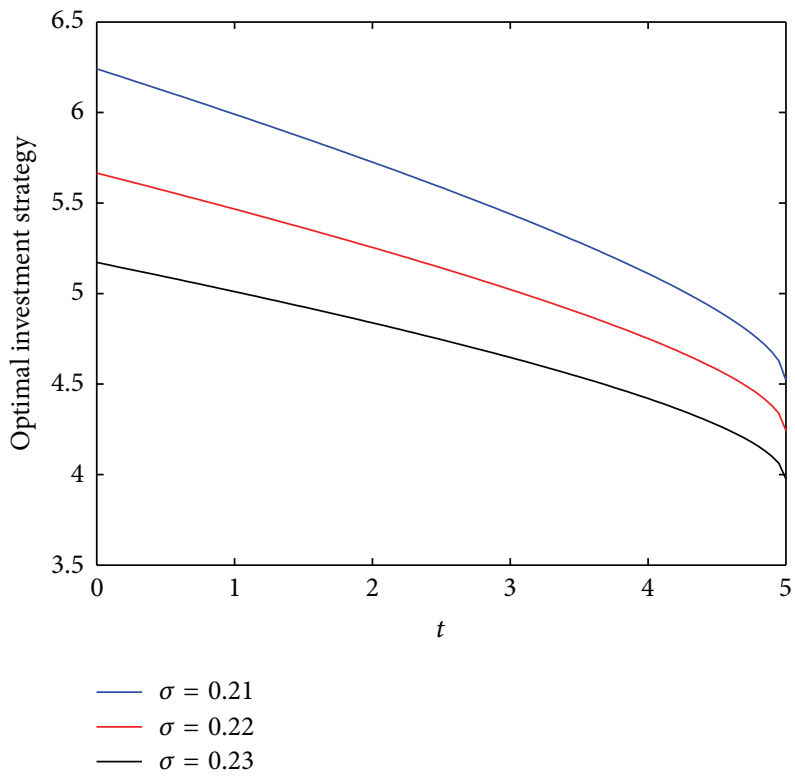

(c) The effect of $\sigma$ on the optimal investment strategy

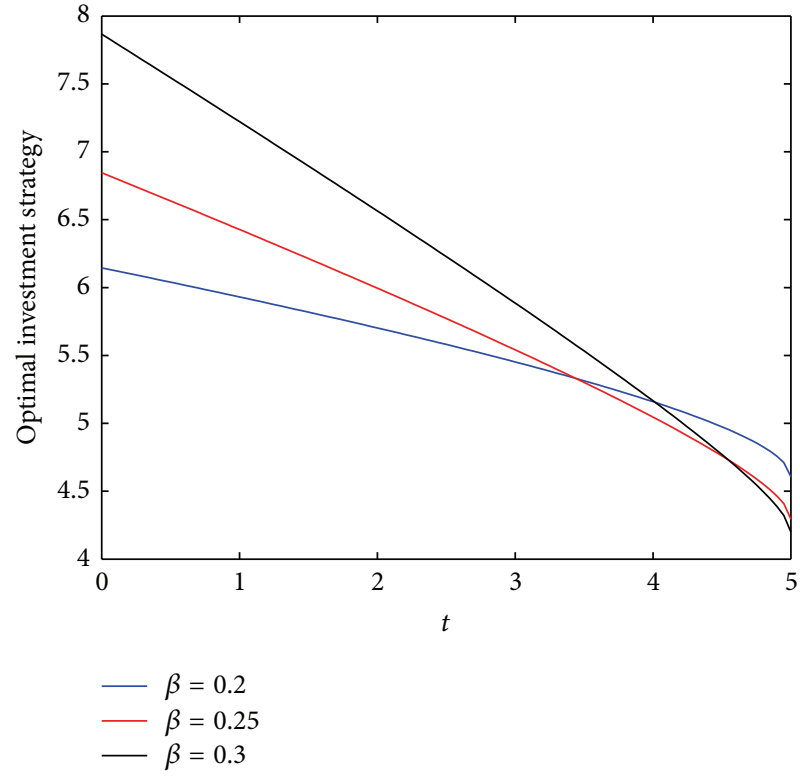

(b) The effect of $\beta$ on the optimal investment strategy

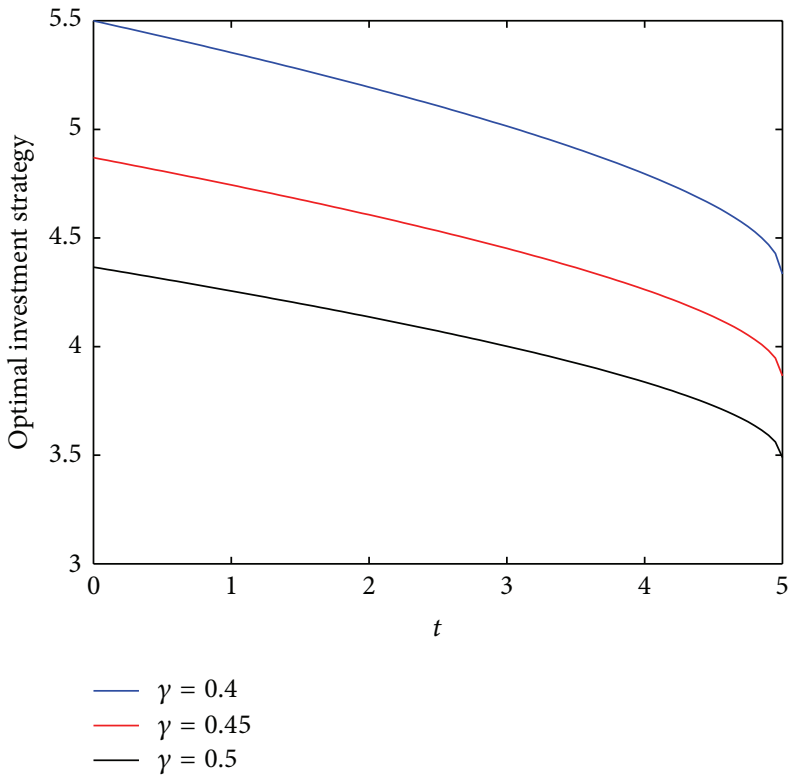

(d) The effect of $\gamma$ on the optimal investment strategy

Figure 2: Effect of $y, \sigma, \beta$, and $\gamma$ on the optimal investment strategy.

Research Funds for the Central Universities (12JNKY002 and 13wkpy28), and Philosophy and Social Science Programming Foundation of Guangdong Province (no. GD12XYJ06).

\section{References}

[1] L. Bai and J. Guo, "Optimal proportional reinsurance and investment with multiple risky assets and no-shorting constraint," Insurance: Mathematics \& Economics, vol. 42, no. 3, pp. 968-975, 2008.

[2] Y. Cao and N. Wan, "Optimal proportional reinsurance and investment based on Hamilton-Jacobi-Bellman equation,"
Insurance: Mathematics \& Economics, vol. 45, no. 2, pp. 157-162, 2009.

[3] C. Irgens and J. Paulsen, "Optimal control of risk exposure, reinsurance and investments for insurance portfolios," Insurance: Mathematics \& Economics, vol. 35, no. 1, pp. 21-51, 2004.

[4] Z. Liang, K. C. Yuen, and J. Guo, "Optimal proportional reinsurance and investment in a stock market with OrnsteinUhlenbeck process," Insurance: Mathematics \& Economics, vol. 49, no. 2, pp. 207-215, 2011.

[5] S. Asmussen, B. Højgaard, and M. Taksar, "Optimal risk control and dividend distribution policies. Example of excessof loss reinsurance for an insurance corporation," Finance and Stochastics, vol. 4, no. 3, pp. 299-324, 2000. 
[6] A. Gu, X. Guo, Z. Li, and Y. Zeng, "Optimal control of excessof-loss reinsurance and investment for insurers under a CEV model," Insurance: Mathematics \& Economics, vol. 51, no. 3, pp. 674-684, 2012.

[7] X. Zhang, M. Zhou, and J. Guo, "Optimal combinational quotashare and excess-of-loss reinsurance policies in a dynamic setting," Applied Stochastic Models in Business and Industry, vol. 23, no. 1, pp. 63-71, 2007.

[8] L. Bai and H. Zhang, "Dynamic mean-variance problem with constrained risk control for the insurers," Mathematical Methods of Operations Research, vol. 68, no. 1, pp. 181-205, 2008.

[9] N. Bäuerle, "Benchmark and mean-variance problems for insurers," Mathematical Methods of Operations Research, vol. 62, no. 1, pp. 159-165, 2005.

[10] Z. Li, Y. Zeng, and Y. Lai, “Optimal time-consistent investment and reinsurance strategies for insurers under Heston's SV model," Insurance: Mathematics \& Economics, vol. 51, no. 1, pp. 191-203, 2012.

[11] Y. Zeng, Z. Li, and Y. Lai, "Time-consistent investment and reinsurance strategies for mean-variance insurers with jumps," Insurance: Mathematics \& Economics, vol. 52, no. 3, pp. 498-507, 2013.

[12] Y. Zeng and Z. Li, "Optimal time-consistent investment and reinsurance policies for mean-variance insurers," Insurance: Mathematics \& Economics, vol. 49, no. 1, pp. 145-154, 2011.

[13] I. Karatzas and H. Wang, "Utility maximization with discretionary stopping," SIAM Journal on Control and Optimization, vol. 39, no. 1, pp. 306-329, 2000.

[14] H. Kraft and M. Steffensen, "Portfolio problems stopping at first hitting time with application to default risk," Mathematical Methods of Operations Research, vol. 63, no. 1, pp. 123-150, 2006.

[15] C. Blanchet-Scalliet, N. El Karoui, M. Jeanblanc, and L. Martellini, "Optimal investment decisions when time-horizon is uncertain," Journal of Mathematical Economics, vol. 44, no. 11, pp. 1100-1113, 2008.

[16] Y. Zeng, H. Wu, and Y. Lai, "Optimal investment and consumption strategies with state-dependent utility functions and uncertain time-horizon," Economic Modelling, vol. 33, pp. 462470, 2013.

[17] I. Kharroubi, T. Lim, and A. Ngoupeyou, "Mean-variance hedging on uncertain time horizon in a market with a jump," Applied Mathematics and Optimization, vol. 68, no. 3, pp. 413444, 2013.

[18] W.-j. Guo and J. Cai, "Portfolio optimization with uncertain exit time in infinite-time horizon," Acta Mathematicae Applicatae Sinica, vol. 29, no. 4, pp. 673-684, 2013.

[19] Z. Yu, "Continuous-time mean-variance portfolio selection with random horizon," Applied Mathematics and Optimization, vol. 68, no. 3, pp. 333-359, 2013.

[20] H. Yao, Y. Lai, and Z. Hao, "Uncertain exit time multi-period mean-variance portfolio selection with endogenous liabilities and Markov jumps," Automatica, vol. 49, no. 11, pp. 3258-3269, 2013.

[21] W. H. Fleming and H. M. Soner, Controlled Markov Processes and Viscosity Solutions, vol. 25 of Applications of Mathematics, Springer, Berlin, Germany, 1993.

[22] A. Pascucci, PDE and Martingale Methods in Option Pricing, vol. 2 of Bocconi \& Springer Series, Springer, New York, NY, USA, 2011.

[23] A. Friedman, Stochastic Differential Equations and Applications, Academic Press, New York, NY, USA, 1975.
[24] J. Grandell, Aspects of Risk Theory, Springer, New York, NY, USA, 1991.

[25] L. Bai, J. Cai, and M. Zhou, "Optimal reinsurance policies for an insurer with a bivariate reserve risk process in a dynamic setting," Insurance: Mathematics \& Economics, vol. 53, no. 3, pp. 664-670, 2013.

[26] I. Karatzas and S. E. Shreve, Brownian Motion and Stochastic Calculus, vol. 113 of Graduate Texts in Mathematics, Springer, Berlin, Germany, 1991. 


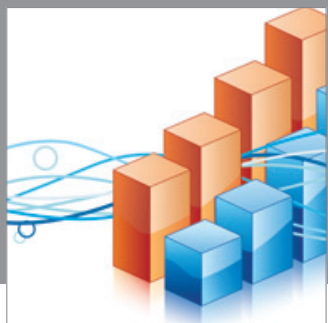

Advances in

Operations Research

mansans

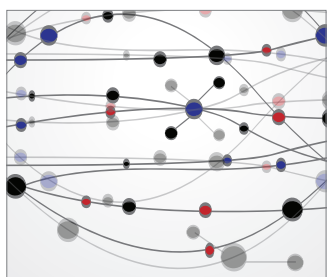

The Scientific World Journal
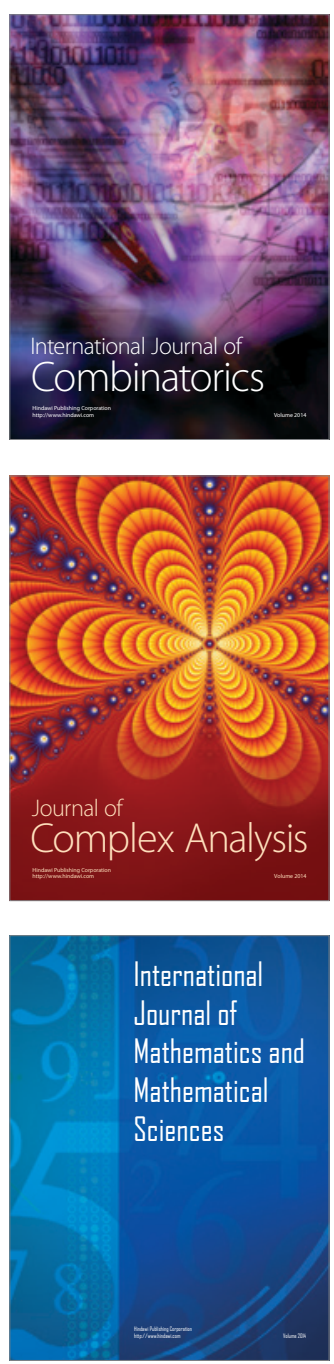
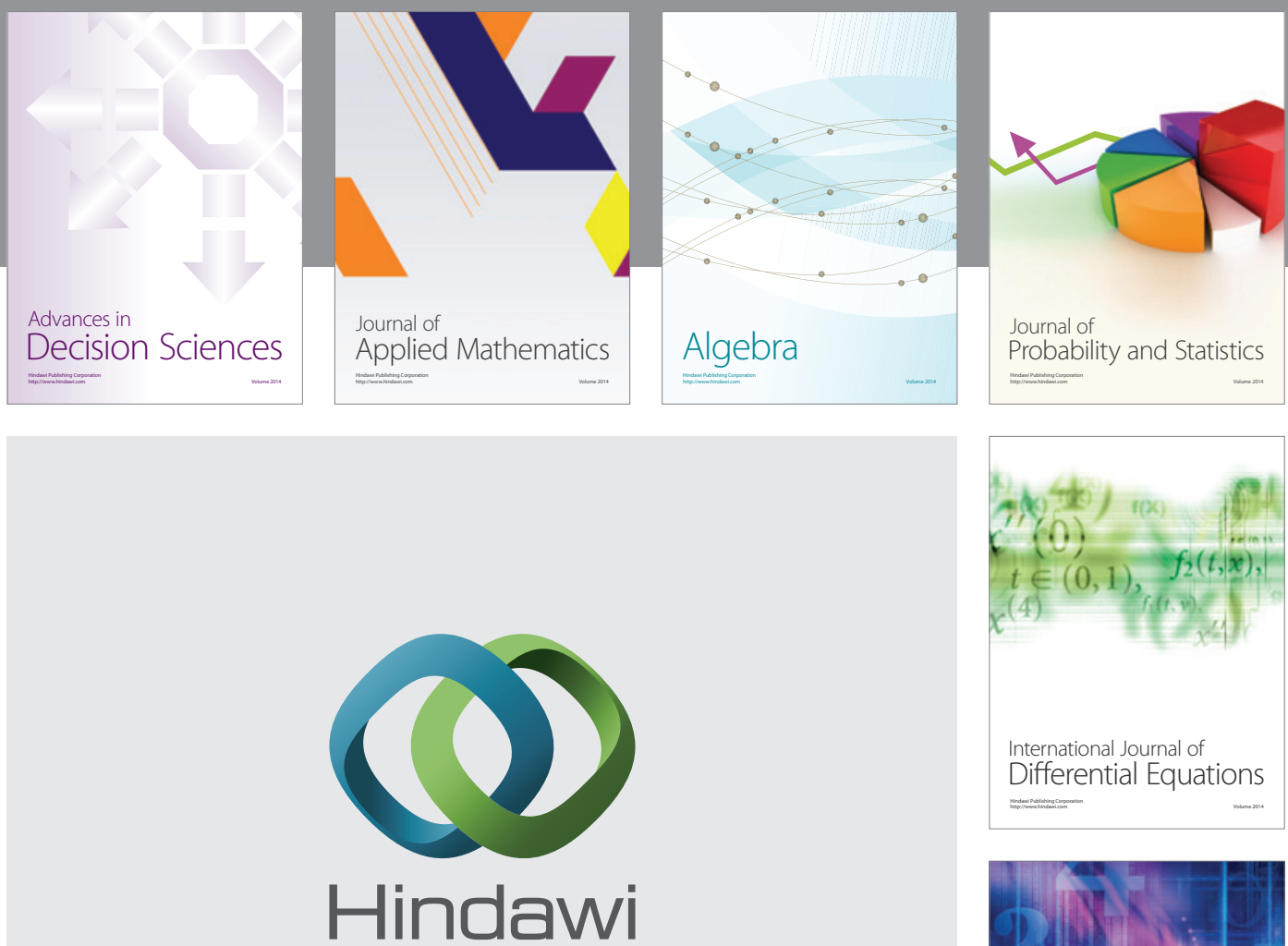

Submit your manuscripts at http://www.hindawi.com
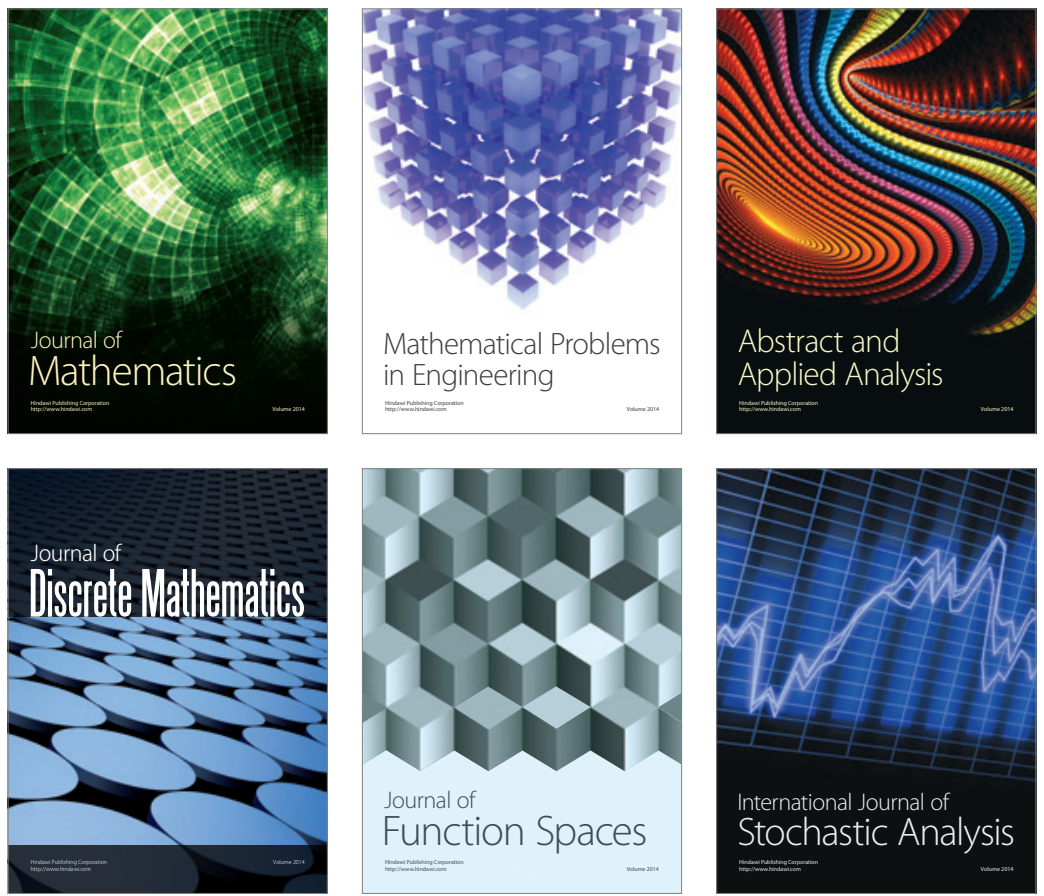

Journal of

Function Spaces

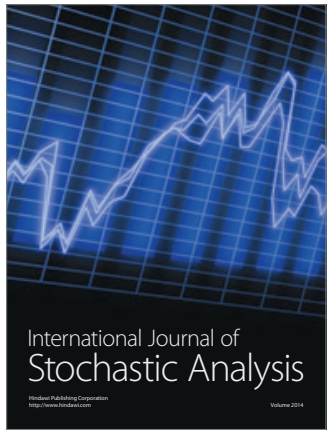

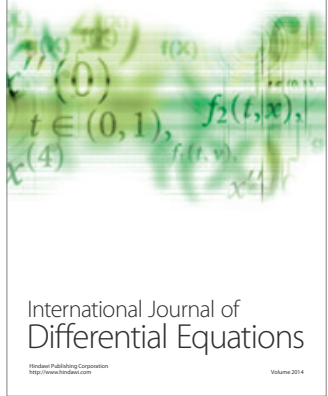
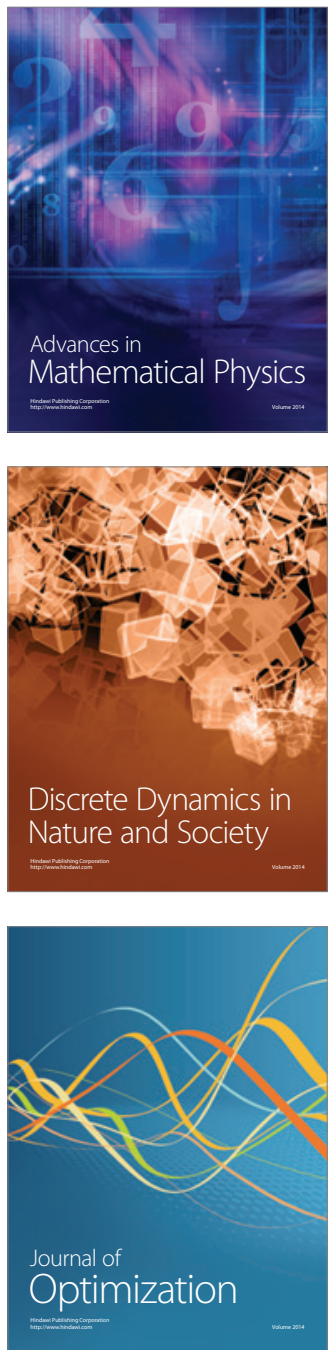